\title{
Correction to: Prospective cohort study of pregnancy complications and birth outcomes in women with asthma
}

\author{
Nasrin Fazel ${ }^{1,2}$ (1) $\cdot$ Michael Kundi $^{1}$ - Erika Jensen-Jarolim ${ }^{3,4} \cdot$ Isabella Pali-Schöll ${ }^{3,4} \cdot$ Asghar Kazemzadeh $^{2}$. \\ Mojtaba Fattahi Abdizadeh ${ }^{5} \cdot$ Habibollah Esmaily $^{6} \cdot$ Roya Akbarzadeh $^{7} \cdot$ Raheleh Ahmadi $^{8}$
}

Published online: 3 December 2018

(c) Springer-Verlag GmbH Germany, part of Springer Nature 2018

\section{Correction to: Archives of Gynecology and Obstetrics (2018) \\ 298:279-287 \\ https://doi.org/10.1007/s00404-018-4800-y}

In the original publication, the university name of the authors in the affiliations was published as University of Medical Sciences, Sabzevar, Iran. The correct university name should read as Sabzevar University of Medical Sciences, Sabzevar, Iran.

The original article can be found online at https://doi.org/10.1007/ s00404-018-4800-y.

Michael Kundi

michael.kundi@meduniwien.ac.at

1 Center for Public Health, Medical University Vienna,

Kinderspitalgasse 15, 1090 Vienna, Austria

2 Department of Internal Medicine, Sabzevar University of Medical Sciences, Sabzevar, Iran

3 Institute for Pathophysiology and Allergy Research, Medical University Vienna, Vienna, Austria

4 The Interuniversity Messerli Research Institute, University of Veterinary Medicine Vienna, Medical University Vienna and University Vienna, Vienna, Austria

5 Department of Microbiology, Sabzevar University of Medical Sciences, Sabzevar, Iran

6 Department of Biostatistics and Epidemiology, Health Research Center, Mashhad University of Medical Sciences, Mashhad, Iran

7 Paramedicine College, Sabzevar University of Medical Sciences, Sabzevar, Iran

8 Department of Obstetrics and Gynecology, Mobini Hospital, Sabzevar University of Medical Sciences, Sabzevar, Iran 